\title{
Design of a Concise and Comprehensive Protocol for Post Stroke Neuromechanical Assessment
}

\author{
Klomp $\mathrm{A}^{1,2 *}$, Van der Krogt JM¹, Meskers CGM${ }^{1}$, De Groot $\mathrm{JH}^{1}$, De Vlugt $\mathrm{E}^{2}$, Van der Helm FCT ${ }^{2}$ and Arendzen $\mathrm{JH}^{1}$ \\ ${ }^{1}$ Leiden University Medical Center, Department of Rehabilitation Medicine, Leiden, Netherlands \\ ${ }^{2}$ Delft University of Technology, Faculty of Mechanical, Maritime and Materials Engineering, Department of Biomechanical Engineering, Delft, Netherlands
}

\begin{abstract}
Functional recovery post stroke is determined by a complex interplay of neural and mechanical (muscular/ tissue) changes. In the present paper, we elaborate on a methodology to assess neuromechanical joint properties in a comprehensive and concise way. A measurement protocol applicable to the wrist joint is introduced and outcome is described for illustrative purposes.

By means of a single axis manipulator, a variety of conditions are applied including different exerted loadings and a passive or active task instruction. The combination of different tasks and loadings systematically excites the nonlinear neuromechanical joint system. Output of the joint system is measured in terms of torques, angular rotation and muscle activation. Both signal analysis and system identification methods are applied to translate the measured variables into physiologically meaningful parameters, describing passive and active (muscle) tissue properties and reflexive characteristics.

A severely impaired and a well-recovered stroke patient show clear differences in outcome parameters. Furthermore, parameters are shown to change over condition, indicating that multiple conditions need to be applied to identify their potentially varying role in movement disorders. The protocol is used in a longitudinal study to explore post-stroke upper limb recovery mechanisms, i.e. the EXPLICIT-stroke study.
\end{abstract}

Keywords: Stroke; Recovery; Arm hand function; Neuromechanics; Assessment

Abbreviations: MVC: Maximal Voluntary Contraction; ECR: Extensor Carpi Radialis; EMG: Electromyography; EXPLICIT-stroke: Explaining Plasticity after Stroke; FCR: Flexor Carpi Radialis; FRF: Frequency Response Function; ROM: Range Of Motion

\section{Introduction}

Movement disorders after stroke may have a major impact on daily life. Almost two thirds of the stroke survivors suffer from sustained deterioration of arm-hand function which threatens physical independency [1]. Besides cortical and corticospinal tract integrity, functional movement and motor deficits are largely determined by joint neuromechanics.

In the acute phase after stroke, mechanical behaviour at joint level is characterized by flaccidity and paresis, while in the sub-acute phase, signs of muscle over-activity and joint stiffening become more prominent $[2,3]$. Although this is a common recovery pattern, several different phenotypes may develop in the chronic phase [4]. These phenotypes will be the result of a complex and varying interplay between neurological and biomechanical changes over time. A better understanding of the interplay and changing contributions of aforementioned neuromechanical processes to movement disorders is needed to address the full functional recovery potential. The EXPLICITstroke (Explaining Plasticity after stroke) study was designed to explore the functional impact of the time-dependent changes in cortical neuroplasticity and neuromechanics, as well as the adaptive compensation strategies that are applied to cope with ischemic brain lesion related motor deficits [5].

Current clinical assessment of joint neuromechanics is restricted to ordinal rating scales such as the Medical Research Council scale for muscle force, goniometry for impaired range of motion (ROM) and Ashworth score for spasticity. The latter however, is incapable of discriminating between the possible neural and/or mechanical sources of increased joint resistance $[4,6,7]$. The use of robotics (e.g. a wrist manipulator) to evoke controlled force and torque perturbations, electromyography (EMG) to record muscle activity and neuromuscular modelling potentially allows for an individual assessment of neurological and biomechanical joint properties [6,8-11].

Nonlinear dynamics of the neuromuscular system greatly influence joint behaviour, yet their role has not been fully recognised. For example, the stretching of tissue yields nonlinear force curves: twice as much stretching does not result in twice as much resistance of the joint [12]. Another example is the sensitivity of the stretch reflexes, which may be modulated at spinal cord level [13]. While linear massspring-damper-like concepts are far easier to apply and are regularly used to simplify mechanical behaviour, they do not comprehensively describe biomechanical properties of the joint under different environmental conditions (tasks and loadings). Using prior knowledge of nonlinearities, the joint can be conditioned such that the nonlinear dynamics of the neuromuscular system can be accounted for, or even parameterized.

In this paper we present the methodological aspects on how

*Corresponding author: A. Klomp MSc, Department of Rehabilitation Medicine, Leiden University Medical Center B0-Q, P.O. Box 9600, 2300 RC Leiden, Netherlands, Tel: 3171 5263457; Fax: 3171 5266697; E-mail: a.klomp@lumc.nl

Received November 29, 2011; Accepted December 26, 2011; Published December 30, 2011

Citation: Klomp A, Van der Krogt JM, Meskers CGM, De Groot JH, De Vlugt E, et al. (2012) Design of a Concise and Comprehensive Protocol for Post Stroke Neuromechanical Assessment. J Bioengineer \& Biomedical Sci S1:008. doi:10.4172/2155-9538.S1-008

Copyright: $\odot 2012 \mathrm{Klomp}$ A, et al. This is an open-access article distributed under the terms of the Creative Commons Attribution License, which permits unrestricted use, distribution, and reproduction in any medium, provided the original author and source are credited. 
to individually address the different properties of the (nonlinear) neurological and biomechanical components of wrist joint behaviour in during flexion-extension movement. This resulted in a comprehensive and clinically applicable assessment protocol. Longitudinal measurements with this specific protocol, within a longitudinal measurement framework such as the EXPLICIT-stroke study, will enhance our knowledge of primary and secondary changes in neuromechanics when functional changes are observed.

\section{Methods}

\section{Line of thought}

Assessment of neurological and biomechanical contributors to movement disorders after stroke should result in structure specific parameters that are potentially modifiable by therapeutic intervention. Treatment is commonly aimed at muscle activation or strength in case of paresis, reduction of reflex sensitivity or neural input in case of hyperreflexia or the stretching of passive tissue in case of joint stiffening. Therefore, we define the neuromechanical system on a therapeutically attainable level into passive, active and reflexive torque components:

- Passive $=$ all joint resistance observed when no neural input is fed to the muscles

- Active $=$ muscle torque generation due to neural input (supraspinal and reflexive)

- Reflexive $=$ active muscle torque solely due to proprioceptive feedback

The interconnection between passive, active and reflexive contributors is represented in Figure 1. By differentiating the contributions of each of these elements to joint level mechanics, their individual roles in movement disorders can be better defined, allowing for targeted therapy.

In order to characterize the phenotype of post-stroke patients properly, the neuromechanical system needs to be sufficiently triggered i.e. different conditions need to be applied. Passive, active and reflexive components will be dependent on the state of the wrist (i.e. joint torque, joint angle and muscle activity) and the externally applied loading. The state represents the current operating point of the system and subsequently its dynamical properties, observed at endpoint level in torque (and angle). A haptic wrist manipulator combined with electromyographic (EMG) measurement is an easy-to-use combination of tools that allows for applying angle or torque controlled perturbations and the subsequent assessment of changes in joint state. The different

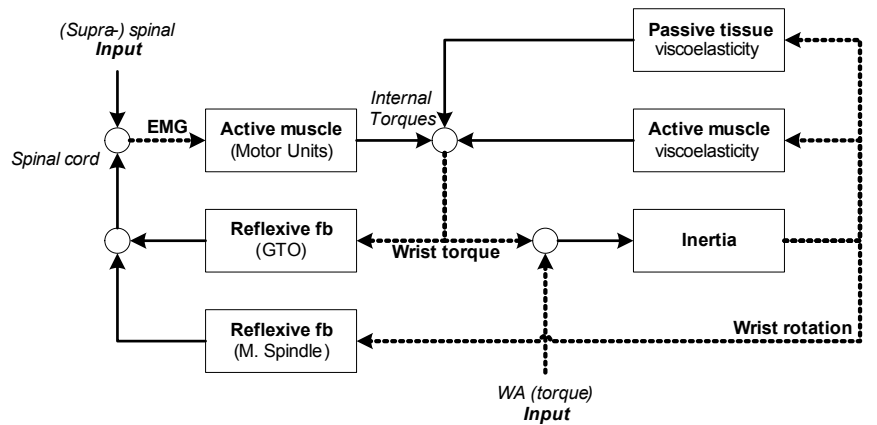

Figure 1: Simplified graphical model of the components of the wrist joint, depicted with active, passive and reflexive elements, corresponding with areas for target therapy. Dotted lines indicate non-invasively measurable connections, EMG (left), torque (center) and rotation (right). Rotation includes angle and rotational velocity. modes in combination with task instruction enable us to impose a desired state. Properties of passive, active and reflexive contributors can be estimated from the measured in- and output data. When torque is the input, angular displacement is the output and vice-versa. Output signals including EMG, as a representative of active muscle state, may also be used to inform the subject on actual task performance.

For analysis, we define two different approaches. The first approach will be referred to as signal analysis. This approach aims to induce large variations regarding the role of model components by applying specific conditions to the system. Slow movements will exclude reflexive activity, while the amount of voluntary contraction can be modulated, and therefore controlled, by proper task instruction. This gives contributor-specific tests that aim to assess passive, active or reflexive contributors individually. The second approach, referred to as system identification, is based on the fact that reflexive resistance is dynamically different from passive or active resistance. Differentiation between the feedback pathways can be done using torque-angle correlation analysis, keeping the closed loop configuration of reflexive (neural) and muscular (mechanical) components into account. Both approaches make it possible to express system performance in terms of its underlying properties, yet conditions are significantly different. System identification methods are not yet sophisticated enough to perform well over a nonlinear domain, and additional signal analysis methods are still needed. Furthermore, limit behaviour, such as ROM or maximal voluntary contraction (MVC), is easier to assess using basic signal analysis. The following subsections describe a listing of interesting outcomes that together result in a comprehensive set for assessment of joint neuromechanics. These outcomes were used as a basis for the protocol discussed in the following section, i.e., "The EXPLICIT-stroke protocol".

\section{Signal analysis}

Passive tests (slow movement while instructed to "do nothing"): Of functional interest are the ROM and the resistance that subjects experience when their joint is moved passively through the ROM. The equilibrium angle of the joint, or rest angle represents a stiffness balance between agonist and antagonistic muscles. Passive tests aim to assess the passive joint structures in subjects, as given in Figure 1. For the assessment of the passive structures, subjects are instructed to do nothing. Movement of the wrist at a slow velocity then results in stretching of passive tissues, while minimizing the contribution of active muscle contraction and reflexive activity. The resulting joint torque will be the result of the stiffness and viscosity arising from predominantly passive contractile and non-contractile tissue. EMG measurements should be used to check for interfering muscle activation during measurement and for data analysis. The following outcomes can be listed for passive tasks:

- Responsive range of motion

- Stiffness and damping

- Rest angle (angle of joint flexion-extension torque equilibrium)

In stroke patients, relative to controls, we expect restrictions in ROM, higher joint stiffness and a rest angle that tends towards flexion [2].

Active tests (slow movement while instructed to "move / push / resist"): These tests address the ability of the patient to actively generate torque at the joint level, preferably in a controlled manner (Figure 1). Applied torque levels should exceed resistance of passive tissue or antagonistic muscles. A subject's ability to generate this particular torque level can be easily tested (also in the clinic) by asking them to flex 
or extend maximally (i.e. the active ROM). Alternative active tests are performed in a standard position (i.e. the rest angle) or during imposed slow movement to minimize reflex activity. Subjects are provided with visual feedback on their actual task performance. Joint angle (relating to overlap of muscle filaments and muscle moment arm, tissue strain) and joint velocity (relating to cross-bridge turnover dynamics and tissue viscosity) also contribute to the potential production of joint torque [14]. Applying an active and a passive test in similar test conditions, defined in terms of joint angle and angular velocity, allows for subtraction of torques generated by the passive structures from the data, under the assumption that the active muscle does not influence stiffness/viscosity values of surrounding passive structures.

From these measurements the following outcomes can be listed for active tasks:

- Self-induced ROM

- Control over joint torque build-up (i.e. quality of motor control)

- Maximally attainable torque

- Angular/velocity dependent joint torque production

In stroke patients, relative to controls, we expect a smaller selfinduced ROM, a lower maximally attainable joint torque and less control over joint torque. Furthermore, it is expected that angulardependency of torque production increases, with an optimum angle (e.g. muscle filament overlap) tending more towards flexion [15].

Reflexive tests (fast movement): Reflexive tests are aimed to assess the reflexive pathways, as given in Figure 1. Higher reflex activity is known to be triggered by high joint angular velocity [11] and together with reflexive time delay (loop-time) it is considered to play an important role in reflex loop stability. To measure the reflexes we commonly use EMG recordings together with controlled, repeated perturbations. This will deliver reproducible data on reflexively triggered muscle activity (e.g. short and long latency reflexes). Perturbations are to be applied at random intervals to minimize anticipation (as subjects can influence their reflexive sensitivity, i.e. reflex modulation). Note however that active components should also be considered when assessing reflexive activity, as functional impairment is defined by the level of joint resistance. In signal analysis methods the possibilities are limited, because the dynamics of reflexively activated muscle are often difficult to distinguish from passive resistance, especially when reflexive activity is small or quickly occurring after perturbation. Clinical measures for spasticity include sudden increase in resistance or EMG and the angle at which this increase occurs, during movement through the ROM (i.e. threshold angle).

These conditions result in the following reflex-related outcomes:

- Reflex loop-time (short and long latency reflex time)

- Reflex magnitude (e.g. area under normalized EMG response)

- Reflexively induced joint torque

- Threshold angle

In stroke patients, relative to controls, we expect a longer shortlatency reflex time, a stronger influence of reflex activity on joint resistance, and a threshold angle located more towards the flexion side of the ROM.

\section{System Identification}

These tests aim at measuring the full joint dynamics in an integral way, while taking the closed loop relation into account. Particular interest lies with the reflexive contributions to joint torque. Continuous random small amplitude torque perturbations induce high velocities and have been proven to allow for the quantification of intrinsic and reflexive components during either a passive or an active (postural control) task [5]. Based on these experiments, active modulation of the reflexive feedback gains, e.g. presynaptic inhibition on muscle spindles and Golgi tendon organs sensory feedback, have been studied using simple linear models. Different signal types include multisines and continuous ramp and hold like perturbations such as ramped block waves or pseudo-random binary sequences. Still the effect of nonlinearity on measurement is unknown and may change over type of perturbation. Clear findings from the wide bandwidth multisine studies were that reflex gains increased with an active task and with the amount of damping provided by the environment.

These integral tests result in the following outcomes:

- Stiffness and damping (of passive plus active structures)

- Reflex loop time

- Reflex magnitude (also referred to as reflex gain: torque contribution to joint dynamics)

\section{- Reflex modulation}

In stroke patients we expect an increased short-latency reflex time, a stronger influence of reflex activity on joint resistance, higher reflex gains, smaller differences between active and passive tasks and less modulation with increase in external damping.

\section{A comprehensive neuromechanical assessment protocol}

A protocol has been set up that assesses passive, active and reflexive components under different conditions, according to the aforementioned line of thought. This protocol is used in the EXPLICIT-stroke study, which aims to assess the relation between primary neural recover and behavioral compensation strategies in arm function recovery after stroke. The measurement set-up comprises a haptic manipulator and EMG-system (Appendix I). Specifications of the set-up have also been validated by Grimaldi et al. [16]. The protocol consists of multiple tests, all of which are either instrumented versions of tests from the clinic or have been tested previously tested on different setups, e.g. Schuurmans et al. [17] on analysis of the reflexive pathway (neural looptime test) and van der Helm et al. [18] and Meskers et al. [19] (amongst others) on multisine perturbations combined with system identification methods. The described neuromechanical protocol uses a combination of tests that all contribute to a post-stroke patient specific signature, and is new in extensively measuring both the structural and functional side of neuromechanical recovery.

\section{Measurement protocol}

The measurement protocol starts with tests which also provide the safety boundaries for later tests (ROM) or feedback target (MVC, restangle). Most tests are applied twice, one for the flexor carpi radialis (FCR) and one for extensor carpi radialis (ECR) muscle activity. Visual feedback of force, position or EMG is provided depending on the task instruction. For maximal active tasks, visual feedback is provided to increase subject motivation. Displayed EMG levels are rectified and averaged over half a second (refresh rate $16 \mathrm{~Hz}$ ). Subjects are allowed to practice. To prevent fatigue, resting time is provided between tests. Total measuring time is approximately $45 \mathrm{~min}$ (including instructions and practice; excluding EMG placement). A full list of used tests and their properties is also given in Table 1 . The analysis of each test is outlined below. After the title of each test an abbreviation is given that refers to the corresponding row in Table 1. 
Citation: Klomp A, Van der Krogt JM, Meskers CGM, De Groot JH, De Vlugt E, et al. (2012) Design of a Concise and Comprehensive Protocol for Post Stroke Neuromechanical Assessment. J Bioengineer \& Biomedical Sci S1:008. doi:10.4172/2155-9538.S1-008

\begin{tabular}{|c|c|c|c|c|c|}
\hline Test & Task & Visual feedback & WA & $\begin{array}{l}\text { WA Reference } \\
\text { signal }\end{array}$ & Parameter \\
\hline ROMP & Passive & none & Torque & $\begin{array}{l}\text { slow increase, } \max \\
2 \mathrm{Nm}\end{array}$ & $P_{\text {ROM }}$ \\
\hline \multirow[t]{2}{*}{ SIR } & passive & none & Angle & $\begin{array}{l}\text { ramp } 0.1 \mathrm{rad} / \mathrm{s} \\
\text { through full ROM }\end{array}$ & $P_{k} P_{d}$ \\
\hline & passive & none & Angle & $\begin{array}{l}\mathrm{ramp} 0.1 \mathrm{rad} / \mathrm{s} \\
\text { through full ROM }\end{array}$ & $P_{R A}$ \\
\hline ROMA & $\begin{array}{l}\text { active: maxi- } \\
\text { mal angular } \\
\text { excursion }\end{array}$ & $\begin{array}{l}\text { attained angles } \\
\text { and current } \\
\text { angle }\end{array}$ & Torque & free (zero torque) & $A_{\text {ROM }}$ \\
\hline MVC & $\begin{array}{l}\text { active: push } \\
\max \end{array}$ & $\begin{array}{l}\text { attained torque } \\
\text { and current } \\
\text { torque }\end{array}$ & Angle & brake & $A_{M V c}$ \\
\hline CJT & active: push & $\begin{array}{l}\text { Increasing } \\
\text { torque target: } \\
\text { ramp } 1 \mathrm{Nm} / \mathrm{s}\end{array}$ & Angle & brake & $A_{C J T}$ \\
\hline \multirow[t]{2}{*}{$\mathrm{ASH}$} & passive & none & Angle & $\begin{array}{l}\text { ramp } 1 \mathrm{sec} \text { through } \\
\text { full ROM }\end{array}$ & $\mathrm{R}_{\mathrm{ta}}$ \\
\hline & passive & none & Angle & $\begin{array}{l}\text { ramp } 0.5 \mathrm{sec} \\
\text { through full ROM }\end{array}$ & $\mathrm{R}_{\mathrm{ta} .5}$ \\
\hline \multirow[t]{2}{*}{$\mathrm{NL}$} & passive & none & Angle & $\begin{array}{l}\text { ramp and hold, } \\
\text { speed } 4 \mathrm{rad} / \mathrm{s} \text {, am- } \\
\text { plitude } 0.14 \mathrm{rad}\end{array}$ & $\mathrm{R}_{\mathrm{It}}$ \\
\hline & $\begin{array}{l}\text { active: push } \\
\text { (const. EMG) }\end{array}$ & $\begin{array}{l}\text { EMG target } \\
\text { level } 10 \% \text { MVC } \\
\text { EMG }\end{array}$ & Angle & $\begin{array}{l}\text { ramp and hold, } \\
\text { speed } 4 \mathrm{rad} / \mathrm{s} \text {, am- } \\
\text { plitude } 0.14 \mathrm{rad}\end{array}$ & $R_{\text {It }}$ \\
\hline \multirow[t]{3}{*}{ WB } & $\begin{array}{l}\text { active: hold } \\
\text { position }\end{array}$ & $\begin{array}{l}\text { Reference angle } \\
\text { incl. history }\end{array}$ & Torque & $\begin{array}{l}\text { crested multisine } \\
0.3-50 \mathrm{~Hz}\end{array}$ & $\begin{array}{l}P_{b-s t}, P_{k-s t}, \\
R_{k v-s t} \\
R_{t d-s}\end{array}$ \\
\hline & passive & $\begin{array}{l}\text { none (EMG for } \\
\text { researcher) }\end{array}$ & Torque & $\begin{array}{l}\text { crested multisine } \\
0.3-50 \mathrm{~Hz}\end{array}$ & $\begin{array}{l}\mathrm{P}_{\mathrm{b}-\mathrm{sl}}, \mathrm{P}_{\mathrm{k}-\mathrm{sl}}, \\
\mathrm{R}_{\mathrm{kv}-\mathrm{sl}} \\
\mathrm{R}_{\mathrm{td}-\mathrm{sl}}\end{array}$ \\
\hline & $\begin{array}{l}\text { active: hold } \\
\text { position }\end{array}$ & $\begin{array}{l}\text { Reference angle } \\
\text { incl. history }\end{array}$ & Torque & $\begin{array}{l}\text { crested multisine } \\
0.3-50 \mathrm{~Hz}+\text { damp- } \\
\text { ing }\end{array}$ & $\begin{array}{l}P_{b-d a}, P_{k-d a} \\
R_{k v-d a} \\
R_{t d-d a}\end{array}$ \\
\hline
\end{tabular}

Table 1: Test specifications of the tests in the EXPLICIT-Stroke protocol including task instruction, visual feedback to the patient Wristalyzer controller mode (WA) Wristalyzer controller reference signal and resulting outcome parameters.

\section{Passive tests (slow movement while instructed to "do nothing")}

1. Range Of Motion Passive - ROMP $\left(\mathrm{P}_{\text {ROM }}\right)$

Applied torques systematically vary between -2 and 2NM. Movement is smoothed by keeping the torque derivative to time low for small torques. The $\mathrm{P}_{\text {ROM }}$ parameter is obtained by taking the difference between the minimal and maximal angle during the ROM test, as given in Figure 2 (left).

\section{Stiffness In Rest - SIR $\left(\mathrm{P}_{R A}, \mathrm{P}_{\mathrm{k}}\right.$ and $\left.\mathrm{P}_{\mathrm{d}}\right)$}

After obtaining the $\mathrm{P}_{\text {ROM }}$, position controlled movement is allowed. The stiffness in rest is tested with a constant velocity, position controlled perturbation. Movement is performed in two directions, resulting in a hysteresis curve $[8,20]$, as shown in Figure 3 . The defined angle of rest $\mathrm{P}_{\mathrm{RA}}$ is taken as the angle where the average hysteresis curve per angle crosses $0 \mathrm{Nm}$ (hence assuming linear damping for small positive and negative velocities). The stiffness and damping related parameters $\left(\mathrm{P}_{\mathrm{k}}\right.$ and $\mathrm{P}_{\mathrm{d}}$ ) are, respectively, the average negative tangent and the average difference of the hysteresis curve over 0.2 rad around the rest angle (Figure 3$)$, divided by the difference in velocity $(0.2 \mathrm{rad} / \mathrm{s})$. The latter will approximate the actual damping if stiffness in both movement directions is equal.

\section{Active tests (slow movement while instructed to "move / push ( resist")}

$$
\text { 3. Range Of Motion Active - ROMA }\left(\mathrm{A}_{\mathrm{ROM}}\right)
$$
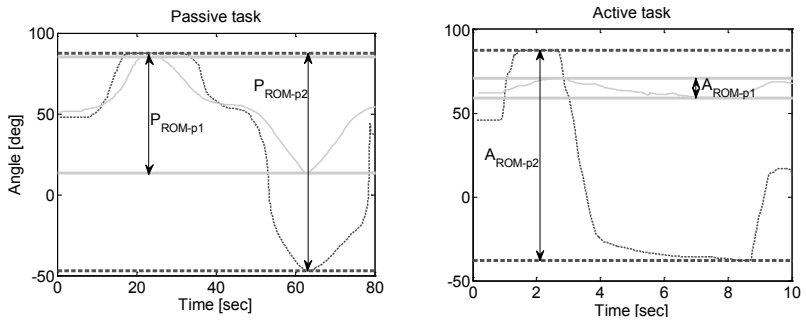

Figure 2: Range of motion data for patient $A$ (light grey, solid - ) and patient $B$ (dark grey, dashed ---). Passive ROM ( $P_{\text {ROM }}$, left) and active ROM ( $A_{R O M}$, right) are indicated with thick horizontal lines.
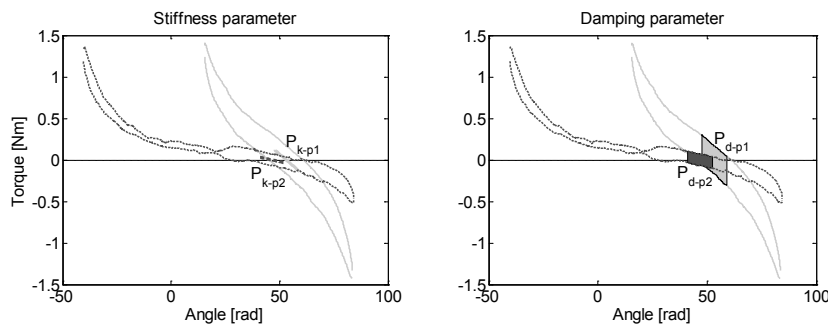

Figure 3: Stiffness in rest test result for patient A (light grey, solid - ) and patient B (dark grey, dashed ---). Thick lines around zero (left figure) indicate average slope of the data at those angles $(\mathrm{Pk})$ and intervals around zero (right figure) show the $(\mathrm{Pd})$
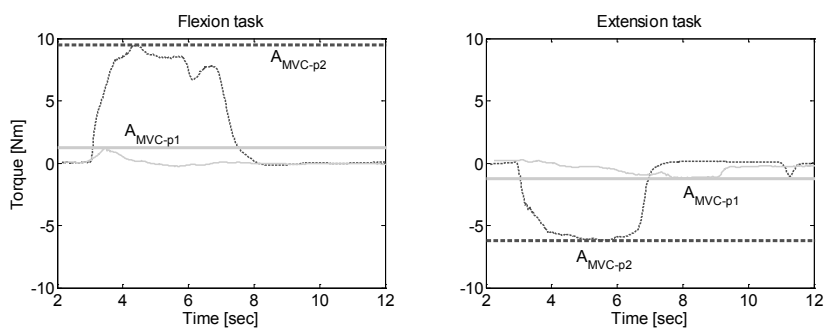

Figure 4: Maximal voluntary contraction data for patient A (light grey, solid -) and patient $B$ (dark grey, dashed ---). Flexion MVC $\left(A_{M V C}\right.$, left) and extension $\operatorname{MVC}\left(A_{\mathrm{MVC}}\right.$, right) are indicated with thick horizontal lines.

For the ROMA test, the Wristalyzer was set to a nonresistant mode. The subject was asked to show his or her maximal ROM. Analysis of the $\mathrm{A}_{\mathrm{ROM}}$ parameter equals the analysis of $\mathrm{P}_{\mathrm{ROM}}$ parameter, given in Figure 2 (right).

\section{Maximal Voluntary Contraction - $\mathrm{MVC}\left(\mathrm{A}_{\mathrm{MVC}}\right)$}

A magnetic break was set and the subject was asked to contract maximally. Torque data was filtered with a $3^{\text {rd }}$ order Butterworth filter of $20 \mathrm{~Hz}$ to reduce the influence of measurement noise. $\mathrm{A}_{\mathrm{MVC}}$ is the maximum measured torque over two repetitions. For clarity, only one dataset (including the $\mathrm{A}_{\mathrm{MVC}}$ ) out of two repetitions has been shown in Figure 4.

\section{Control over Joint Torque - CJT $\left(\mathrm{A}_{\mathrm{CIT}}\right)$}

The Control over Joint Torque (CJT) test is added to address the quality of motor control of the subject. The magnetic break was applied and the subject was asked to steadily increase his or her level of contraction $(1 \mathrm{Nm} / \mathrm{s})$. Torque data was again filtered for analysis with a $3^{\text {rd }}$ order Butterworth filter of $20 \mathrm{~Hz}$ to reduce influences measurement noise. The level at which the patient fails to follow the reference torque $\left(\mathrm{A}_{\mathrm{CJT}}\right)$ is determined by the point at which the patient generated torque 

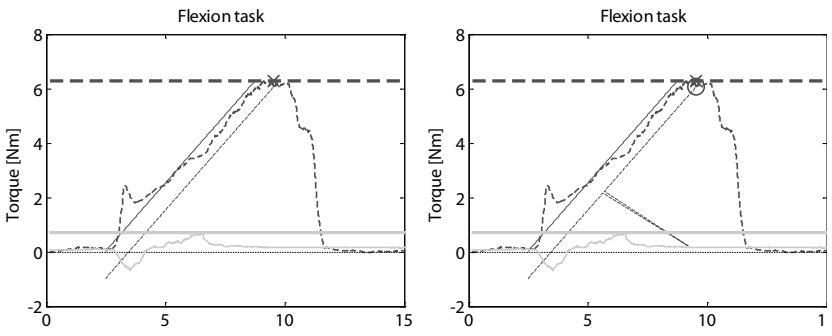

Figure 5: Control over Joint Torque data ACJT for patient A (light grey, solid - ) and patient $B$ (dark grey, dashed ---), for extension (left) and flexion (right). The diagonal straight solid line indicates the reference force over time, the diagonal dashed line shows the maximal follow lag. Circles indicate where the patient fails to follow the reference torque; parameters $A_{C J T}$ for patient $B$ are indicated with a cross.
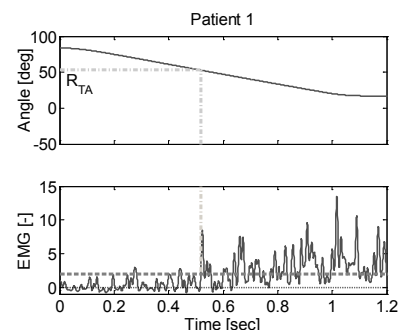
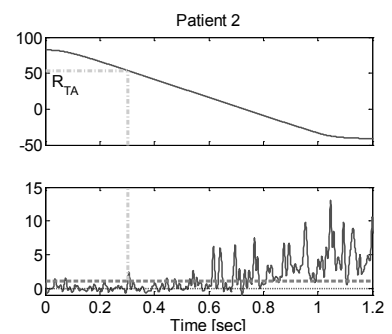

Figure 6: Ashworth data of patient A (left) and patient B (right). Measured FCR EMG data (dark solid lines -), 2.81 times the standard deviation of the background (dashed lines ---) and the derivation of the threshold angle parameter Rta (dashed-dotted lines -.-.) are shown. Both figures represent the condition of $1 \mathrm{ROM} / \mathrm{sec}$ angular velocity and movement towards extensions.

is lower than $1 \mathrm{Nm}$ under the reference torque (circle in Figure 5). The maximal force during the test (thick horizontal line in Figure 5) is used as an upper boundary for $\mathrm{A}_{\mathrm{CJT}}$.

\section{Reflexive tests (fast movement)}

\section{Ashworth-ASH $\left(\mathrm{R}_{\mathrm{ta}}\right.$ and $\left.\mathrm{R}_{\mathrm{ta} .5}\right)$}

An instrumented version of the clinical modified Ashworth test has been included in the protocol to address the reflexive system. The Ashworth test gives insight into the reflexive properties during a large sweep through the ROM. Moreover, it allows for validation of the use of an Ashworth test in the clinic.

For the analysis, the EMG signal is rectified and filtered with a $3^{\text {rd }}$ order low pass Butterworth filter of $80 \mathrm{~Hz}$. We first determined the angle for which the EMG signal exceeds 5 times the standard deviation of the background EMG (horizontal dotted line in Figure 6). The preceding point where the EMG signal exceeds 2.81 times the standard deviation (i.e. resulting in $0.25 \%$ probability of being background signal if normally distributed) is taken as the onset of the EMG response to the perturbation. The corresponding angle at that onset time is the threshold angle $\left(\mathrm{R}_{\mathrm{ta}}\right)$.

\section{Neural Looptime-NL $\left(\mathrm{R}_{\mathrm{lt}}\right.$ and $\left.\mathrm{R}_{\text {auc } \_\mathrm{M1}}\right)$}

The neural looptime tests consist of nine ramps ( $2 \mathrm{rad} / \mathrm{s})$ and hold $(0.75 \mathrm{~s})$ perturbations that occur at random time intervals. After the hold phase a returning phase using a minimal jerk profile is initiated. For the analysis, the data of each of the runs is averaged over the nine perturbations to reduce noise. This sequence is performed in a passive and active condition; first the subject is asked to relax, second to push against the handle with constant EMG at $10 \%$ of measured MVC
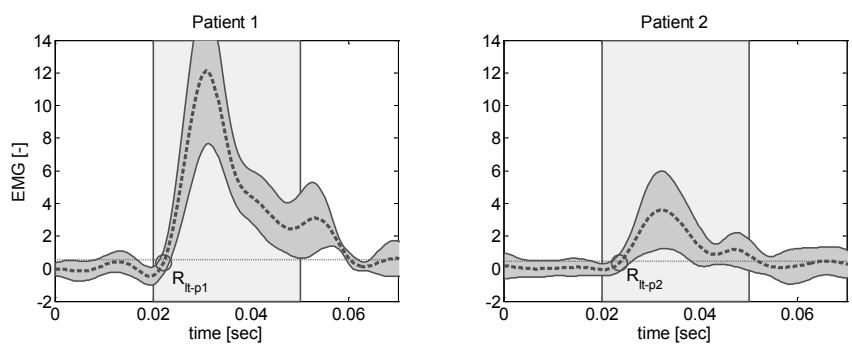

Figure 7: Neural looptime test datasets for patient A (left) and patient B (right). Average and standard deviation of nine FCR EMG responses (mean: dashed line ---, standard deviation: grey surrounding surface) and corresponding analysis interval and values $\left(R_{\mathrm{tt}}\right.$ : circle $O$ and $R_{\text {AUC }}$ : surface under --- within the indicated interval $[0.02-0.05] \mathrm{sec})$. Dotted line $(\cdots)$ indicates the 2.81 times the standard deviation of the background EMG.
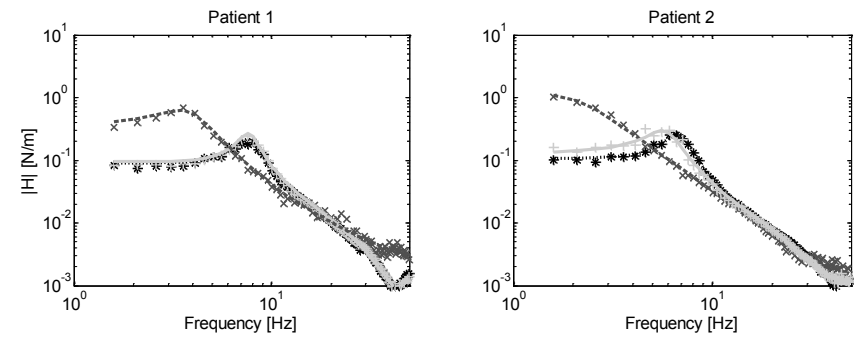

Figure 8: Bode magnitude plot of multisine-test data from patient $A$ (left) and $B$ (right). Bode magnitude representation of the model fits of the data for the 3 measurement conditions: stiff (fit: dotted $\cdots$, FRF estimate: *), slack (fit: dashed ---, FRF estimate: $\mathrm{x}$ ) and damp (fit: solid line -, FRF estimate: +).

EMG. For the latter rectified and filtered EMG (averaging 8 times/ $\mathrm{sec}$ ) is shown to the subject as a vertical bar with a target area at $10+/$ $2.5 \%$ for the stretched muscle. Color of the bar changed from red to green when entering the target area. Subjects were instructed to ignore perturbations as much as possible and to return to the target area after perturbations, when necessary. Figure 7 shows the resulting average EMG signal with its cross-trial standard deviation. The EMG signal is rectified and filtered with a $3^{\text {rd }}$ order low pass Butterworth filter of $80 \mathrm{~Hz}$. The base level (BL) of the EMG is determined as the average EMG over the time window $[-400,-20] \mathrm{ms}$ with respect to the start of the perturbation [17]. The EMG signal $\mathrm{E}$ is then normalized to the signal $\mathrm{E}_{\mathrm{n}}$ using the baselevel BL:

$$
\mathrm{E}_{\mathrm{n}}(\mathrm{t})=\mathrm{E}(\mathrm{t}) / \mathrm{BL}-1
$$

Reflexive EMG response to the input stretch is assumed to be significant if the signal exceeds 2.81 times the standard deviation of the baselevel, indicated with the horizontal dotted line in Figure 7. The short-latency reflex onset $\mathrm{R}_{\mathrm{lt}}$ and area under the curve $\mathrm{R}_{\text {auc } \mathrm{M} 1}$ are then obtained (time window for both parameters limited to $[20,50] \mathrm{ms}$, with respect to pulse onset [17]).

\section{System Identification}

8. Multisine perturbations-WB $\left(\mathrm{P}_{\mathrm{k}}, \mathrm{P}_{\mathrm{d}}, \mathrm{R}_{\mathrm{td}}, \mathrm{R}_{\mathrm{kv}-\mathrm{st}}, \mathrm{A}_{\mathrm{k}-\text { act }}, \mathrm{A}_{\mathrm{d}-\mathrm{act}}\right.$ $\mathrm{R}_{\mathrm{rm}-\mathrm{act}}$ and $\mathrm{R}_{\mathrm{rm} \_ \text {env }}$ )

A set of multisine perturbations has been added to address the subjects' capabilities in disturbance rejection and adaptation to a changing environment (increased damping). Multisine perturbations represent a functional disturbance and may be supported by findings from the neural looptime test. 
Possible low frequent compensational behavior of the patient (against drift away from the target angle), will be corrected for by first pre-filtering the raw data using a $3^{\text {rd }}$ order butterworth high pass filter of $1 \mathrm{~Hz}$. A frequency response function (FRF) is then estimated using a closed loop estimator on the pre-filtered data,

$$
\mathrm{H}_{\mathrm{FRF}}=\mathrm{S}_{\mathrm{da}} / \mathrm{S}_{\mathrm{dT}}
$$

Where $S_{\mathrm{da}}$ and $\mathrm{S}_{\mathrm{dT}}$ are the estimated cross spectral densities of the externally applied disturbance torque $\mathrm{d}$ with the measured angle a and interaction torque T, using a Fast Fourier Transform [18].

The resulting FRF is then averaged in groups of 8 frequency points to improve the estimate. Finally, a model $[18,19]$, based on the block scheme depicted in Figure 1 is fitted to the acquired FRF (Figure 8). Inertia, spring $\left(\mathrm{P}_{\mathrm{k}<\text { condition }}>\right)$, damper $\left(\mathrm{P}_{\mathrm{d}<\text { condition }}>\right)$, eigenfrequency of muscle activation, velocity dependent reflex gain $\left(\mathrm{R}_{\mathrm{kv} \_<\text {condition }}>\right)$, reflex speed $\left(\mathrm{R}_{\mathrm{td}<\text { condition }}>\right)$ and grip dynamics parameters are all included in the model [18]. Different types of reflexive feedback (position, velocity and force) were tested, yet only velocity feedback gave low parameter variability, in accordance with earlier results of Meskers et al. [19]. Microneurographic studies imply velocity feedback is muscle spindle feedback, as opposed to Golgi Tendon Organ feedback [21]. Using different conditions (stiff, slack and damp, see Table 3) the changes of the system over condition can be investigated. The difference in stiffness and damping between passive and active conditions yields the activation induced stiffness and damping $\mathrm{A}_{\mathrm{k}_{\mathrm{a}} \text { act, }} \mathrm{A}_{\mathrm{d} \_ \text {act }}$ :

$$
\begin{aligned}
& A_{k \_a c t}=A_{k_{\_} s t}-A_{k \_s l} \\
& A_{d \_a c t}=A_{d \_s t}-A_{d \_s l}
\end{aligned}
$$

Difference in reflex gain over conditions results in the modulation parameters Rrm_act and Rrm_env:

$$
\begin{aligned}
& \mathrm{R}_{\mathrm{rm} \_a c t}=\mathrm{R}_{\mathrm{kv} \_s t}-\mathrm{R}_{\mathrm{kv} \_s l} \\
& \mathrm{R}_{\mathrm{rm} \_ \text {env }}=\mathrm{R}_{\mathrm{kv} \_d a}-\mathrm{R}_{\mathrm{kv} \_s t}
\end{aligned}
$$

\section{Results}

\begin{tabular}{|c|c|c|c|c|}
\hline & Parameter & Parameter & Patient A & Patient B \\
\hline \multirow[t]{2}{*}{ Passive } & ROM Passive & $\mathrm{P}_{\mathrm{ROM}}$ & $\begin{array}{l}72 \text { deg } \\
{[13,6-85,1]}\end{array}$ & $\begin{array}{l}135 \mathrm{deg} \\
{[-46,9-87,7]}\end{array}$ \\
\hline & Restangle & $P_{R A}$ & 53 deg flexion & 34 deg flexion \\
\hline \multirow[t]{3}{*}{ Active } & ROM Active & $\mathrm{A}_{\mathrm{ROM}}$ & $\begin{array}{l}17 \mathrm{deg} \\
{[49,1-65,9]}\end{array}$ & $\begin{array}{l}126 \text { deg } \\
{[-38,1-87,7]}\end{array}$ \\
\hline & $\begin{array}{l}\text { Maximal Voluntary Contrac- } \\
\text { tion (MVC) at rest angle }\end{array}$ & $A_{M v c}$ & $\begin{array}{l}\text { F: } 1,2 \mathrm{Nm} \\
\mathrm{E}: 1,3 \mathrm{Nm}\end{array}$ & $\begin{array}{l}\text { F: } 9,5 \mathrm{Nm} \\
\mathrm{E}: 6,2 \mathrm{Nm}\end{array}$ \\
\hline & $\begin{array}{l}\text { Control over Joint Torque } \\
\text { (CJT) }\end{array}$ & $A_{\text {CJT }}$ & $\begin{array}{l}\text { F: } 0 \mathrm{Nm} \\
\mathrm{E}: 0 \mathrm{Nm}\end{array}$ & $\begin{array}{l}\mathrm{F}: 6,2 \mathrm{Nm} \\
\mathrm{E}: 3,2 \mathrm{Nm}\end{array}$ \\
\hline \multirow[t]{3}{*}{$\begin{array}{l}\text { Reflex- } \\
\text { ive }\end{array}$} & Reflexive Looptime & $\mathrm{R}_{\mathrm{It}}$ & $\begin{array}{l}F: 22 \mathrm{~ms} \\
E: 32 \mathrm{~ms}\end{array}$ & $\begin{array}{l}F: 24 \mathrm{~ms} \\
\mathrm{E}: 23 \mathrm{~ms}\end{array}$ \\
\hline & Area under the M1 curve & $R_{\text {auc_m1 }}$ & $F: 0,16 \mathrm{E}: 0,01$ & $\begin{array}{l}\text { F: } 0,05 \\
E: 0,05\end{array}$ \\
\hline & Threshold angle & $\mathrm{R}_{\mathrm{ta}}$ & $\begin{array}{l}F: 53 \mathrm{deg} \\
\mathrm{E}:-\end{array}$ & $\begin{array}{l}F: 55 \mathrm{deg} \\
\mathrm{E}:-\end{array}$ \\
\hline
\end{tabular}

Parameter values taken from two stroke patients are given in Table 2 and 3, for illustration purposes. Both patients A and B are female (aged 55 and 45, respectively) and were measured more than one year post-stroke. Ashworth scores for the measured and impaired left

\begin{tabular}{|c|c|c|c|c|}
\hline & Parameter & Parameter & Patient A & Patient B \\
\hline \multirow[t]{2}{*}{ Passive } & Stiffness at Restangle & $P_{k}$ & $2,8 \mathrm{Nm} / \mathrm{rad}$ & $1,0 \mathrm{Nm} / \mathrm{rad}$ \\
\hline & Damping at Restangle & $P_{d}$ & $0,07 \mathrm{Nms} / \mathrm{rad}$ & $0,05 \mathrm{Nms} / \mathrm{rad}$ \\
\hline \multirow[t]{2}{*}{ Active } & $\begin{array}{l}\text { Additional activation } \\
\text { induced muscle stiffness } \\
\text { at Restangle }\end{array}$ & $A_{k_{-} \text {act }}$ & $8,3 \mathrm{Nm} / \mathrm{rad}$ & $8,3 \mathrm{Nm} / \mathrm{rad}$ \\
\hline & $\begin{array}{l}\text { Additional activation } \\
\text { induced muscle damping } \\
\text { at Restangle }\end{array}$ & $A_{d_{-} \text {act }}$ & $0,08 \mathrm{Nms} / \mathrm{rad}$ & $0,08 \mathrm{Nms} / \mathrm{rad}$ \\
\hline \multirow[t]{4}{*}{ Reflexive } & Reflexive Looptime & $R_{\text {td st }}$ & $30 \mathrm{~ms}$ & $29 \mathrm{~ms}$ \\
\hline & $\begin{array}{l}\text { Reflexive contributions to } \\
\text { joint resistance }\end{array}$ & $\mathrm{R}_{\mathrm{kv} \_s t}$ & $0,080 \mathrm{Nms} / \mathrm{rad}$ & $0,092 \mathrm{Nms} / \mathrm{rac}$ \\
\hline & $\begin{array}{l}\text { Reflex modulation due to } \\
\text { activation }\end{array}$ & $R_{\mathrm{m} \_ \text {act }}$ & $0,06 \mathrm{Nms} / \mathrm{rad}$ & $0,06 \mathrm{Nms} / \mathrm{rad}$ \\
\hline & $\begin{array}{l}\text { Reflex modulation due to } \\
\text { environmental changes }\end{array}$ & $R_{m_{-} \text {env }}$ & $0,01 \mathrm{Nms} / \mathrm{rad}$ & $-0,06 \mathrm{Nms} / \mathrm{rad}$ \\
\hline
\end{tabular}

Table 2: Parameters following from the signal analysis technique tested on a paretic chronic stroke patient (pt. A) and a functionally recovered chronic stroke patient (pt. B). E refers to Extension and F to Flexion. All angles, except for ROM parameters, are given in degrees from the zero angle of the Wristalyzer.
Table 3: Parameters following from the system identification technique tested on a paretic chronic stroke patient (pt. A) and a functionally recovered chronic stroke patient (pt. B)

wrist joint of both patients were manually determined to be 3 and 0 respectively.

\section{Passive parameters}

Patient A shows a smaller passive ROM $\left(\mathrm{P}_{\mathrm{ROM}}\right)$ and an increased stiffness $\left(\mathrm{P}_{\mathrm{k}}\right)$ and viscosity $\left(\mathrm{P}_{\mathrm{d}}\right)$ at the restangle, both for large and small perturbations, as shown in Table 2 and 3, respectively. This indicates a higher resistance against movement from passive structures, as muscle activation is checked for using EMG. Differences between Table 2 and Table 3 values are expected as they differ greatly in condition. The data underlying the system identification method contains only small angular deviations, while the data underlying the signal analysis is performed during a long sweep through the ROM. Earlier research on passive stiffness has shown that in the initial phase of movement the stiffness is larger [12]. Furthermore, the rest angle $\left(\mathrm{P}_{\mathrm{RA}}\right)$ of patient $\mathrm{A}$ is located more towards flexion (Table 2). It is known that in the subacute phase the restangle moves towards flexion [2].

\section{Active parameters}

In Table 2, the combination of a decreased active ROM ( $\left.\mathrm{A}_{\mathrm{ROM}}\right)$ and a low MVC $\left(\mathrm{A}_{\mathrm{MVC}}\right)$ is seen in the resulting parameters of patient A. Movement is determined by a both the potential voluntary torque and the stiffness of the joint, for each angle. Therefore we can expect a smaller value for the $\mathrm{A}_{\mathrm{ROM}}$ parameter given low voluntary contraction (e.g. $\mathrm{A}_{\mathrm{MVC}}$ in the restangle) and stiffness of passive structures (e.g. $\mathrm{P}_{\mathrm{k}}$ in the restangle). Control over Joint Torque $\left(\mathrm{A}_{\mathrm{CJT}}\right)$ values show the amount of joint torque control in a slowly increasing tracking task. $A_{C T T}$ values are expected to be slightly lower than the MVC and are found to be approximately $60 \%$ of the MVC value for patient $\mathrm{B}$. The $\mathrm{A}_{\mathrm{CTT}}$ is set to zero for patient $A$ as the maximal force in test does not exceed $1 \mathrm{Nm}$ (see appendix II for more details on analysis). Table 3 coincidentally shows equal values for stiffness $\left(\mathrm{A}_{\mathrm{k}_{\mathrm{n}} \text { act }}\right)$ and damping $\left(\mathrm{A}_{\mathrm{d}_{\mathrm{a} a c t}}\right)$, induced by the "hold position" task instruction. This shows that both patients were able to increase their joint stiffness voluntarily, yet the control over the unilaterally produced joint torque $\left(\mathrm{A}_{\mathrm{CTT}}\right)$ is a lot lower for patient $\mathrm{A}$.

\section{Reflexive parameters}

The reflex loop time $\left(\mathrm{R}_{\mathrm{lt}}\right)$ of about $23 \mathrm{~ms}$ is comparable to the values found in literature [17]. The higher value for patient A was due to low reflexive activity for that condition $\left(\mathrm{R}_{\mathrm{m} 1 \text { _auc }}\right)$. The looptime parameter resulting from the system identification procedure (Table $3, \mathrm{R}_{\mathrm{td} \_\mathrm{st}}$ ) is 
generally higher. This difference is expected to be of methodological nature; for system identification procedures the best fit may lie more towards the long latency response, instead of the short latency response onset. Still, the two are assumed to be equal in their physiological background and do not yield additional information. For patient B the area under the curve Rm1_auc is similar for FCR and ECR. The Rm1_auc value for the ECR of patient A is substantially lower than the other values, indicating a very small reflex, if at all present. The FCR muscle shows a very high response, possibly indicating hyperreflexia. Additional looptime $\left(\mathrm{R}_{\mathrm{lt}}\right)$ and reflexive activity $\left(\mathrm{R}_{\text {auc m1 }}\right)$ are believed to contribute to joint instability. Threshold angles $\left(\mathrm{R}_{\mathrm{ta}}\right)$ are similar for both patient $\mathrm{A}$ and $\mathrm{B}$ (the flexion angle prior to movement is with 85 degrees also equal for both patients, see $\mathrm{P}_{\text {ROM }}$, Table 1). The angular velocities at which these were determined differ significantly (72deg/sec and $135 \mathrm{deg} / \mathrm{sec}$ for patient A and B, respectively), which, as reflexes are velocity dependent, explains the quick response of the more recovered subject. Threshold angles are only given for the FCR muscle as the stretching of the ECR muscle did not result in reflexive activation. Reflexive contributions to joint resistance $\left(\mathrm{R}_{\mathrm{kv} \text { stiff }}\right)$ are stronger in patient B. For both patients activation of the muscles has a large influence on the modulation of reflexes $\left(\mathrm{R}_{\mathrm{rm} \text { _act }}\right)$. Furthermore, a more stable, damped environment does not trigger reflex modulation $\left(\mathrm{R}_{\mathrm{rm} \text { env }}\right)$ for patient A and unexpectedly [19] causes negative reflex modulation for patient $\mathrm{B}$.

\section{Discussion}

We presented a methodology that can be used to obtain insight into the potential roles of passive, active and reflexive contributors to movement disorders. Using a combination of test conditions and available analysis methods it is possible to discriminate different contributors of the movement disorder. We used this methodology to design a protocol that can be used to assess the role of neuromechanics in stroke recovery, to be used in the EXPLICIT-stroke study. This specific protocol is responsive to changes in severity of movement disorder. Passive parameters show altered stiffness, active parameters show paresis and diminished control. Reflexive parameters show altered reflex gain, loop time and modulation. Furthermore, differently conditioned tests have shown different values for the same parameter, potentially resulting in an altered role in joint dynamics for that specific contributor.

Compared to current, manual clinical tests such as the Ashworth test, a full protocol might seem cumbersome. However, a combination of different conditions or tests is required to determine the major contributors to a movement disorder. The presented protocol, in combination with a haptic manipulator (the Wristalyzer), is comprehensive yet concise, safe and non-invasive.

With the current setup it is not possible to discern continuous involuntary active tissue from passive viscoelastic properties during passive tasks. Furthermore, specifically for some of the active tests in the EXPLICIT-stroke protocol, a certain amount of independent control of agonist and antagonist muscles is required, which some patients will not be able to perform. However, these tests can still give valuable results. The issued parameters reflect the properties of a highly non-linear system. As such, presented parameters are to be considered a mean over e.g. a movement trajectory. Non-linear identification techniques should be developed to assess instantaneous determinant characteristics during true functional tasks. Furthermore, there are some indications that patients particularly have trouble adapting from one state to the next [19], as opposed to attaining that new state at all.
Adding time variance (e.g., in task instruction or virtual environments) during tests could highlight differences between stroke patients and healthy subjects.

The primary outcome measures will be validated in a larger group of chronic patients versus a control group of healthy subjects. Furthermore, longitudinal analysis of changes in neuromechanics in acute and subacute stroke patients will be conducted in the EXPLICITstroke program [5], a program targeted at understanding the relation of primary neural repair to behavioural compensation in arm function recovery. This multicenter research project comprises the longitudinal assessment of parameters of functional recovery (clinimetrics), recovery of cortical activation (fMRI), intactness of corticospinal tract (TMS) and compensation strategies (kinematics). Neuromechanics describe the integration of neural and muscle activation, determining actual functional performance. As such, the assessment of neuromechanics within the EXPLICIT-stroke study is essential in understanding the relation between primary (neural) lesion and functional performance.

\section{Conclusion}

A comprehensive, yet concise assessment protocol was developed to identify tissue, active muscular and reflexive properties at joint level. Application of the protocol illustrates the necessity to apply multiple measurement and task conditions to identify patients within a spectrum of neuromechanical system functions and to ultimately understand the underlying mechanism of a movement disorder after neural lesions.

\section{Appendix I: Measurement Setup}

Based on the earlier work on wrist manipulators [22] a torque controlled haptic wrist manipulator has been developed, called the Wristalyzer $囚$, (MOOG Nieuw Vennep, the Netherlands) [16] in collaboration with the Delft University of Technology and the Leiden University Medical Center (Figure 9). The setup consists of a main drive of a vertically positioned servo motor (Parker SMH100 series). The arm is fixated to the Wristalyzer and the hand is fixated to the handle. The motor axis is aligned with the rotation axis of the wrist joint. Movement of the motor is therefore directly coupled to flexion/extension of the wrist. The handle has an ellipsoidal shape which, because of its length, prevents finger flexion (Figure 9).

\section{Wristalyzer}

- Handle $R O M \approx 180^{\circ}$, accuracy $0.35^{\circ}$

- Nominal motor torque is $6 \mathrm{Nm}$

- Magnetic break torque $20 \mathrm{Nm}$

- Maximal angular velocity $2000 \mathrm{deg} / \mathrm{s}$ [16]

- Encoder (Hiperface absolute single turn)

- Strain gauge (mounted between the axis and handle)

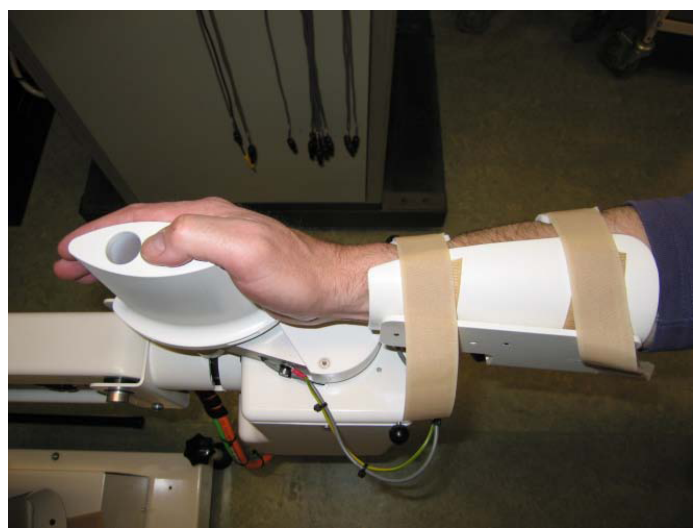

Figure 9: Top-view of the Wristalyzer®, (MOOG, Nieuw Vennep, the Netherlands). 
Citation: Klomp A, Van der Krogt JM, Meskers CGM, De Groot JH, De Vlugt E, et al. (2012) Design of a Concise and Comprehensive Protocol for Post Stroke Neuromechanical Assessment. J Bioengineer \& Biomedical Sci S1:008. doi:10.4172/2155-9538.S1-008

\section{EMG}

Muscle activation was measured using a Delsys Bagnoli-8 system with bipolar surface electrodes on the Flexor Carpi Radialis (FCR) and two on the Extensor Carpi Radialis (ECR).

\section{Measurement computer}

A laptop is used for data processing and visualization. Matlab ${ }^{\circ}$ R2007a was used for communication with the Wristalyzer and Matlab® R2008b was used for data analysis.

\section{Acknowledgments}

This work was supported by grants from ZonMW (grant 89000001), het Revalidatiefonds, Revalidatie Nederland and VRA, and is part of the EXPLICITStroke project. The authors would like to acknowledge René van Ee and David C. Balderas Silva, who substantially contributed to the development of the measurement software.

\section{References}

1. Dobkin $\mathrm{BH}(2005)$ Clinical practice. Rehabilitation after stroke. N Engl J Med 352: 1677-1684.

2. Gracies JM (2005) Pathophysiology of spastic paresis. I: Paresis and soft tissue changes. Muscle Nerve 31: 535-551.

3. Gracies JM (2005) Pathophysiology of spastic paresis. II: Emergence of muscle overactivity. Muscle Nerve 31: 552-571.

4. Dietz V, Sinkjaer T (2007) Spastic movement disorder: impaired reflex function and altered muscle mechanics. Lancet Neurol 6: 725-733.

5. Kwakkel G, Meskers CG, van Wegen EE, Lankhorst GJ, Geurts AC, et al. (2008) Impact of early applied upper limb stimulation: the EXPLICIT-stroke programme design. BMC Neurol 8: 49

6. Fleuren JF, Voerman GE, Erren-Wolters CV, Snoek GJ, Rietman JS, et al (2010) Stop using the Ashworth Scale for the assessment of spasticity. J Neurol Neurosurg Psychiatry 81: 46-52.

7. Pandyan AD, Johnson GR, Price Cl, Curless RH, Barnes MP, et al. (1999) A review of the properties and limitations of the Ashworth and modified Ashworth Scales as measures of spasticity. Clin Rehabil 13: 373-383.

8. Pisano F, Miscio G, Del Conte C, Pianca D, Candeloro E, et al. (2000) Quantitative measures of spasticity in post-stroke patients. Clin Neurophysiol 111: 1015-1022.
9. Mirbagheri MM, Barbeau H, Kearney RE (2000) Intrinsic and reflex contributions to human ankle stiffness: variation with activation level and position. Exp Brain Res 135: 423-436.

10. Sinkjaer T, Hayashi R (1989) Regulation of wrist stiffness by the stretch reflex. J Biomech 22: 1133-1140.

11. De Serres SJ, Milner TE (1991) Wrist muscle activation patterns and stiffness associated with stable and unstable mechanical loads. Exp Brain Res 86: 451 458 .

12. Axelson HW, Hagbarth KE (2001) Human motor control consequences of thixotropic changes in muscular short-range stiffness. J Physiol 535: 279-288.

13. Stein RB (1995) Presynaptic inhibition in humans. Prog Neurobiol 47: 533-544.

14. Rassier DE, MacIntosh BR, Herzog W (1999) Length dependence of active force production in skeletal muscle. J Appl Physiol 86: 1445-1457.

15. Bialocerkowski A, Grimmer KA (2003) Measurement of isometric wrist muscle strength--a systematic review of starting position and test protocol. Clin Rehabi 17: 693-702.

16. Grimaldi G, Lammertse P, van den Braber N, Meuleman J, Manto M (2008) A new myohaptic device to assess wrist function in the Lab and in the Clinic-the Wristalyzer. Eurohaptics 5024: 33-42.

17. Schuurmans J, de Vlugt E, Schouten AC, Meskers CG, de Groot JH, et al (2009) The monosynaptic la afferent pathway can largely explain the stretch duration effect of the long latency M2 response. Exp Brain Res 193: 491-500.

18. van der Helm FC, Schouten AC, de Vlugt E, Brouwn GG (2002) Identification of intrinsic and reflexive components of human arm dynamics during postural control. J Neurosci Methods 119: 1-14.

19. Meskers CG, Schouten AC, de Groot JH, de Vlugt E, van Hilten BJ, et al. (2009) Muscle weakness and lack of reflex gain adaptation predominate during poststroke posture control of the wrist. J Neuroeng Rehabil 6: 29.

20. Dunne JW, Singer BJ, Allison GT (2003) Velocity dependent passive muscle stiffness. J Neurol Neurosurg Psychiatry 74: 283

21. Matthews PB (1981) Evolving views on the internal operation and functiona role of the muscle spindle. J Physiol 320: 1-30.

22. Schouten AC, de Vlugt E, van Hilten JJ, van der Helm FC (2006) Design of a torque-controlled manipulator to analyse the admittance of the wrist joint. $J$ Neurosci Methods 154: 134-141. 